\title{
Rasgos de la ontología fundamental
}

\author{
Edward Javier Ordóñez*
}

\section{Resumen}

El objetivo de este ensayo es presentar los rasgos fundamentales de la ontología fundamental de Martin Heidegger. En el desarrollo se puntualizará la radicalidad del pensamiento en la diferencia ontológica. La primera, corresponde al carácter fenomenológico de la ciencia, es decir, a la búsqueda husserliana de una ciencia estricta; la segunda, a los resultados de la investigación ontológica hecha por Heidegger y presentados en Ser y tiempo. La radicalidad conducirá a la búsqueda del fundamento de la ontología y al mismo tiempo al carácter originario de la pregunta por el ser. Si bien la radicalidad no es un rasgo fundamental de la ontología fundamental, da lugar a la primera característica: el dasein, entendido como los logros obtenidos en la tematización de la pregunta por el ser; es decir, de la búsqueda por el sentido general del ser que se encontrará en el ente con primacía óntico-ontológica.

Palabras clave: Ontología fundamental, analítica existenciaría, diferencia ontológica, ser y tiempo.

\section{Traits Fundamental Ontology}

\section{Abstract}

The aim of this paper is to present the main features of the fundamental ontology of Martin Heidegger. In the development of thought points radicalism in the ontological difference: the first corresponds to the phenomenological nature of science, that is, to the search of a strict science Husserl, the second corresponds to the results of research conducted by Heidegger ontological and presented in Being and Time. In other words, the radical lead to the search for the foundation of ontology and at the same time, the originating status of the question of being, however, it is not a feature of fundamental ontology, open to the first feature, the ontological difference: Dasein. Understood as those achievements in the theming of the question of being, i.e. the search for the general sense of being to be found in the body with ontic-ontological primacy.

Keywords: Fundamental ontology, existential analysis, ontological difference, being and time

\footnotetext{
* Magíster en Filosofía por la Benemérita Universidad Autónoma de Puebla (México). Doctorante en Estudios Latinoamericanos, Universidad Nacional Autónoma de México. Becario del Consejo Nacional de Ciencia y Tecnología de México (CONACYT). E-mail: edward.javier11@gmail.com
} 


\section{Introducción}

La expresión "ontología fundamental" recoge el ideario filosófico de Heidegger, o bien aquella intención de posibilitar la metafísica en su fundamento, que a su vez influye en su obra temprana. Ontología fundamental permea de varias maneras lo publicado en Ser y tiempo, al punto de confundirse con la analítica existenciaria. "De ahí que la ontología fundamental -escribe Heidegger- que está a la base de todas las otras ontologías, deba ser buscada en la analítica existenciaria del dasein" (Heidegger, 1927, p. 23). Aunque la analítica existenciaría comprenda un estudio exhaustivo del dasein y de este como comprensión del ser, no es cierto que desarrolle y agote la ontología fundamental. La analítica existenciaria es un momento -quizá el primero- de la problemática presente en la expresión.

Se acusa a la ontología fundamental de reduccionista al extremo de confundirla con antropología filosófica. Reduccionista, en cuanto la gradación heideggeriana del mundo comprende únicamente a los entes intramundanos, al ente a la mano y al dasein; antropología filosófica, porque se creyó fue usada exclusivamente para definir al hombre y las investigaciones filosóficas latinoamericanas así lo demuestran. Su base antropológica promueven ese énfasis: “¿Cuál sería el carácter del hombre dentro de la historia? [...] el hombre es, en su existencia, un constante hacerse, un proyecto y comprensión de la posibilidad de desarrollarse" (Magallón, 1991, p. 131). Sin embargo, se puede argüir que para Heidegger ambas críticas obedecen a tematizaciones de orden no necesario; es decir, la ontología fundamental al preguntarse por el "fundamento" puede referir este tipo de elementos epistémicos, pero de ello no se exige su necesaria explicitación.

Del mismo modo, se rescata la ontología fundamental como teoría del conocimiento en cuanto prepondera la "constitución finita del ser del hombre como condición de posibilidad de toda ciencia y disciplina, sea teórica o práctica" (García, 2002, p. 127). Pero la ontología fundamental se levantó en contra del neokantismo en el sentido de hacer de las ideas kantianas un asunto de teoría del conocimiento. La crítica de Heidegger se centraba en que los neokantianos explicaban el carácter de cientificidad de alguna disciplina desde el aspecto lógico; es decir, privilegiaron la lógica trascendental por encima de la estética trascendental. Heidegger hizo un giro en las interpretaciones: ya no era la lógica trascendental sino la estética trascendental la que determinaba, en cierto sentido, la cientificidad de cualquier teoría.

En esta época -escribe García- Heidegger mantenía aún viva la idea de Husserl sobre la cientificidad (wis- senchaftlichkeit) de la filosofía, pero refiriéndose con ello no a una filosofía científica en el sentido neokantiano o positivista del término, sino comprendiendo a la "fenomenología como la verdadera ontología científica" (García, 2002, p. 127).

Sin embargo, aunque es positivo el hecho de rescatar los avances de la ontología fundamental respecto a otras teorías de la época, ello no es suficiente para tildarla de teoría del conocimiento. Con todo, el carácter privilegiado en este ensayo refiere que el presupuesto de la ontología fundamental es el fundamento de la metafísica, que, al mismo tiempo, en términos de Ser y tiempo refiere la pregunta por el ser.

\section{La radicalidad en Heidegger}

Una característica de la ontología fundamental -quizá la primera- reposa en la radicalidad del pensar heideggeriano. El carácter fenomenológico de Heidegger nace en la discusión con Aristóteles, Husserl, Dilthey y las escuelas neokantianas de Maburgo y Baden, quienes posibilitaron la radicalidad del pensar en Heidegger ya que con una sensibilidad filosófica propia miraron problemas en la filosofía tradicional. ¿Por qué se adjetiva de radical a la filosofía de Heidegger? O ¡en qué medida una visión como la de Heidegger renueva una lectura de Aristóteles? Esbozando una respuesta a la segunda, quizá se pueda responder a la primera.

La filosofía de Aristóteles tenía el carácter de universal; en "pensar lo que es en tanto es" radica su universalidad. Además, remitía al ser supremo; era teología. El problema radica en ese punto, en que es una ciencia particular (tiene como objeto a Dios) y al mismo tiempo, es una ciencia universal $-o$ al menos esa es su pretensión- ya que mira al ente en cuanto ente. Para el problema, Jaeger propuso una solución a partir de un trabajo genético. Heidegger propuso una solución más radical: trató de actualizar a Aristóteles incluso en la misma traducción. Heidegger cuestionó los conceptos heredados por la tradición filosófica y los actualizó hasta el punto de darle una nueva interpretación. En este sentido Xolocotzi-Yáñez (2010) posiciona la actitud filosófica de Heidegger. En su libro Una crónica de ser y tiempo de Martin Heidegger, lo destaca subrayando una carta suya a Elfride:

[...] la fuerza primordial de la vida sobrepasa al conocimiento en profundidad y plenitud; toda nuestra filosofía está enferma, justamente porque sus problemas se determinan a partir de lo que ya ha sido conocido y juzgado, de tal manera que 
son desfigurados y se convierten en paradojas (Heidegger, 2008, citado por Xolocotzi-Yánez, 2010, p. 59).

La filosofía primera es una episteme; es primera -o mejor aún, originaria- no por alguna explicación acerca de la propiedad del objeto, sino por el carácter universal en cuanto necesidad.

La ciencia originaria es una ciencia preteorética en cuanto ha elaborado las estructuras fundamentales de cada "región esencial" (las regiones, ya sean de cosas o esenciales refieren -según Rivera- diferenciaciones dentro del todo de los entes. Existe una diferencia entre las expresiones "regiones de cosas" y "regiones esenciales": la primera es una primera distinción espontánea y natural que no exige mayor explicación o cuidado; por ejemplo, el sector de los entes no vivos y el sector de los vivientes. La segunda "surge de un descubrimiento y de una delimitación de un carácter más preciso y, en definitiva, relación con los distintos saberes que se vuelcan sobres esas regiones esenciales, ya sea la filosofía, ya las distintas ciencias" (Rivera \& Stuven, 2008, pp. 54-55), no gracias a la investigación científica, sino "a la experiencia e interpretación precientífica del dominio de ser que define la región esencial misma” (Heidegger, 1927 , p. 20). Es decir, en la ciencia original hay una elaboración previa y no teorética del ser; es una experiencia vital de las cosas. El carácter preteorético/"preontológico" refiere una interpretación vital (auslegung). La discusión entra la naturaleza de la filosofía y la ciencia conlleva una discusión más problemática y por ende más radical, ya que la filosofía primera va a referir el ámbito de comprensión, mientras que la ciencia indica el ámbito lógico. En fin, se puede afirmar la radicalidad de Heidegger ante la postura aristotélica y con esta permear la ontología fundamental, al parafrasear lo dicho Xolocotzi-Yánez (2010) en la primera sesión del seminario Aproximaciones al giro (kehre) en la filosofía del Martin Heidegger: Jaeger fue el filólogo, Heidegger el filósofo. Al respecto, Leo Strauss propone otro testimonio:

I remember the impression he made on me when I heard him first as a Young Ph.D., in 1922. Up to that time I had been particularly impressed, as many of my contemporaries in Germany were, by Max Weber: by his intransigent devotion to intellectual honesty, by his passionate devotion to the idea of science- a devotion that was combined with a profound uneasiness regarding the meaning of science. On my way north from Freiburg, where Heidegger then taught, I saw, in Frankfurt-am-Main, Franz Rosenzweig, whose name will always be remember when informed people speak about existentialism, and I told him of Heidegger. I said to him that, in comparison with Heidegger, Weber appeared to me as an "orphan child" in regard to precision and probing and competence. I had never seen before such seriousness, profundity, and concentration in the interpretation of philosophic texts. I had Heard Heidegger's interpretation of certain sections in Aristotle, and sometime later O Heard Werner Jaeger in Berlin interprets the same texts. Charity compels me to limit my comparison to the remark that there was no comparison (Strauss, 1989, pp. 27-28).

Por su parte, la pregunta por el ser es "radical" en cuanto a su primacía, metodología y conclusiones, ya que la metafísica, según Heidegger, al perder su fundamento exige nuevas explicitaciones. Ella posee primacía ontológica y óntica; escribe Heidegger: "¿O es, por el contrario, la pregunta más fundamental y a la vez la más concreta?" (Heidegger, 1927, p. 19). Primacía ontológica, porque trasciende el investigar temático dado en el interior de las ciencias. Las ciencias no deben considerar su legitimidad en lo que respecta a resultados acumulados. Por el contrario, su valor se medirá al ser capaces de considerar sus "conceptos fundamentales". No obstante, tal tarea debe ser previa al carácter científico; es decir, dicha investigación fundamentará la pretensión de cientificidad. El método científico impide una discusión rigurosa acerca de sus fundamentos, por tanto las ciencias poseen limitaciones a la hora de establecer para sí mismas el carácter de cientificidad. La filosofía, por el contrario, puede acercarse y discurrir sobre aquellos conceptos fundamentales de la ciencia; puede contribuir al carácter científico. Ella legitima la ciencia gracias a su carácter poietico/creativo y Heidegger lo denomina "lógica productiva" (Heidegger, 1927, p. 21; Rivera \& Stuven, 2008, p. 60), puesto que abre el mundo de los conceptos fundamentales. La filosofía trata sobre ellos; empero, no puede dejar de indagar acerca del ser en general; si así lo fuera, la investigación sería incompleta. Por lo tanto, la primacía ontológica de la pregunta por el ser aparece en la medida en que ayuda a fundamentar el carácter científico de las ciencias y al mismo tiempo, en cuanto que indaga por el sentido general del ser. Una argumentación similar antecede la cuestión por la primacía óntica y sus respectivas conclusiones:

La pregunta por el ser apunta a determinar las condiciones $a$ priori de la posibilidad no sólo de las ciencias que investigan el ente en cuanto tal o cual, y que por ende se mueven ya siempre en una comprensión del ser, sino que ella apunta también a determinar la condición de posibilidad de las ontologías mismas que anteceden a las ciencias ónticas y las fundan (Heidegger, 1927, p. 21).

Entonces, ¿por qué la pregunta por el ser es radical? Porque ella responde a la exigencia husserliana de "la filosofía como ciencia rigurosa o estricta" y al mismo tiempo, porque en ella prima la fundamentación de la 
filosofía como ciencia originaria que tiene por objeto la vida (leben). "Concretamente, lo que Heidegger busca es una esfera originaria (Ursprungsgebiet) en la vida humana fáctica, un aspecto de ella que haga posible una consideración originaria, pero de carácter estrictamente científico" (Lambert, 2008, p. 86). No obstante, en la radicalidad no reposa la primera característica de la ontología fundamental; por el contrario, la permite. La radicalidad admite ganar claridad en cuanto a la diferencia ontológica. Por tanto, la primera característica de la ontología fundamental es la diferencia ontológica. Dicho de otra manera: Heidegger se inserta en las raíces de la metafísica tradicional para buscar su fundamento; lo encuentra en el ser, pero "el ser" ha sido olvidado (Seinsvergessenheit) por la tradición filosófica occidental: ella se ha detenido en sus categorías quiditativas. Para Heidegger es necesario el ser y el modo de acceso al él, el ente. Ser-ente, según Navarro-Cordón (1966), es la categoría dual que está en el centro de la ontología fundamental y con su explicitación, la superación de la metafísica.

Con esto ha quedado seńalado el sentido y el camino a recorrer por la ontología fundamental. Por ser un saber del "fundamento" ha de versar sobre él en cuanto ser y verdad. Pero por ser un "saber", se ha de hacer necesariamente cuestión del único ente al que es dado la compresión del ser, el dasein. Un estudio originario de ambos fundamentos llevará a cabo la superación de la metafísica, que no es en definitiva sino la tematización de la diferencia ontológica (Navarro-Cordón, 1966, p. 31).

\section{El ente es. El ser se despliega}

La pregunta por el ser exige la tematización de la diferencia ontológica. ¿Cómo lo hace Heidegger? Primero, comprende la pregunta como "búsqueda", ya que en ese sentido posee una comprensión de aquello que busca: el ser. "Todo preguntar es una búsqueda" (Heidegger, 1927, p. 15). Segundo, explica las estructuras fundamentales de la pregunta, dado que permiten aclarar el tipo de pregunta de este "preguntar". Él no es un "simple preguntar"; es decir, no es un sin saber lo que se hace al preguntar, sino un cuestionamiento explícito. Así, cuestionar la diferencia ontológica cumple con la estructura constitutiva de la pregunta: das Gefragte, das Befragte y das Erfragte. La primera, refiere lo puesto en cuestión; la segunda, lo interrogado; y la última, lo propiamente preguntado. De manera precisa, lo puesto en cuestión - das Gefragte- es el sentido del ser (Heidegger, 1989). De él, Heidegger afirma que se posee cierta comprensión: "no sabemos lo que significa ser. Pero ya cuando preguntamos: ¿qué es ser?, nos movemos en una comprensión del es [...] Esta comprensión del ser mediana y vaga es un factum" (Heidegger, 1927, p. 16).

La comprensión mediana -también comprensión primaria- es positiva porque posee diversas indeterminaciones del ser. Empero, la pregunta por el ser deberá conceptualizarlo de manera precisa. Por tanto, el "ser" que se tiene que elaborar, según Heidegger, es "aquello que determina al ente en cuanto ente" (Heidegger, 1927, p. 17). De esta manera, el ente corresponderá a lo interrogado, das Befragte, ya que lo preguntado, el "ser", es entendido como ser del ente. "El ente será interrogado, por así decirlo, respecto de su ser" (Heidegger, 1927, p. 17).

Por su parte, lo propiamente preguntado, das Erfragte, corresponderá al sentido del ser, ya que lo puesto en cuestión, el ser en general, no puede ser comprendido con el concepto de presencia. Mejor aún, ya que el ser no es presencia inmediata el interrogante se dirigirá a su sentido. En fin, hasta aquí se puede afirmar que la ontología fundamental consiste en determinar la estructura del ente en el que se da la comprensión del ser. No obstante, en ello no se agota la tematización de la diferencia ontológica. Ella también exige la analítica del dasein, ya que según NavarroCordón, "sólo en la caracterización fenomenológica de sus constitutivos dinámicos, podrá recogerse desde la unidad su validez ontológica" (Navarro-Cordón, 1966, p. 33).

Ahora bien, el ente es lo interrogado. Pero hay diversas formas de entes y no todas dan cuenta rigurosamente de lo que es el ser. Entonces, pregunta Heidegger: ¿¿n cuál ente se debe leer el sentido del ser, desde cuál ente deberá arrancar la apertura del ser? En el dasein; el "ente que somos en cada caso nosotros mismos,y que, entre otras cosas, tiene esa posibilidad de ser que es el preguntar" (Heidegger, 1927, p. 18). La analítica del dasein intentará mostrar cómo este ente posee primacía óntico-ontológica; óntico, en cuanto el dasein existe; somos nosotros mismos y somos existentes, fuera de ello no hay más; ontológica, porque este ente está determinado por la existencia misma y es capaz de comprenderla y con esta viene una comprensión de todo lo que le concierne; es decir, una comprensión ontológica del mundo. "El dasein es para sí mismo ónticamente 'cercanísimo', ontológicamente lejanísimo y sin embargo, preontológicamente no extraño" (Heidegger, 1927, pp. 26-27).

De esta manera, se puede apreciar cómo la analítica del dasein pretenderá un análisis preparatorio del ser del hombre como posibilidad única de todas las ontologías, como ontología fundamental; empero, ella no puede supervalorar o desvalorizar algún elemento constitutivo. Tal analítica, al tratar de corresponder a la imparcialidad 
propia de la metodología fenomenológica, se detendrá en su "cotidianidad media" (Alltäglichkeit). El recurso de la "cotidianidad" evitará, en cierta medida, el carácter problemático del modo de acceso a este ente, dado que ella, según Rivera en una nota explicativa de su traducción de Ser y tiempo "describe al dasein en la forma como se da inmediatamente en la vida cotidiana, previa a toda teoría y a toda filosofía. La cotidianidad media es, por así decirlo, el modo corriente y ordinario como el dasein se vive a sí mismo" (Heiddegger, 1927, p. 423).

El dasein en referencia a la primacía óntico-ontológica es existencia y está determinado por ella. Pero, ¿cuál es el sentido de "existencia"? ¿Qué sentido tiene decir que el hombre existe? La existencia (existenz), en su sentido etimológico, debe ser entendida como ex-sistencia, es decir, como salida afuera o estar afuera. Ex-sistir es "sobrepasar la realidad simplemente presente en dirección de la posibilidad" (Vattimo, 1987, p. 18). Y dado que ella determina el ser del hombre, la existenz es el modo propio de ser del dasein; el ex-sistir propio del hombre va en contravía de lo pensado en la ontología tradicional; es decir, la existencia no es la simple presencia (vorhandenheit). Dasein es ex-sistencia; está en el horizonte de la posibilidad. En definitiva, dasein es poder-ser. El dasein es constituido en cuanto ex-sistencia, en cuanto poder-ser, en cuanto abierto, y ello corresponde al significado del da. Por otra parte, dado que el ex-sistir se da como modo constitutivo del dasein, refiere una correlación intrínseca que impide pensarlo como un ente más; impide pensarlo sin mundo; es decir, al carácter ontológico del dasein, poder-ser/da, le corresponde el mundo. Ya no es válida la pregunta por el mundo como un afuera o un adentro; no estoy en el mundo como aquello que está afuera de mí; el dasein corresponde a ser-en-el-mundo.

Entonces, ¿qué es el mundo? La fenomenología del mundo indica, en primer lugar, que él no es la totalidad de las cosas; antes bien, él es la condición para que ellas aparezcan. "Gracias a él podemos movernos libremente en nuestro uso con ellas" (Held, 1997, p. 15). Para Husserl, el mundo como totalidad solo es posible en la articulación de los horizontes individuales en uno universal; para Heidegger, la totalidad del mundo se da en el mismo horizonte de uso (zuhandenheit). No obstante, el mundo no se agota en la finitud; ella ha adquirido un sentido completamente nuevo; con ella el mundo pasa a ser una dimensión del dasein, un modo de ser del estar abierto. Por tanto, el mundo no tiene carácter de ente, sino carácter ontológico. En segundo lugar, la mundanidad del mundo se funda solo en la base del dasein. Lo que refiere que en la raíz del darse del mundo aparece el dasein. La experiencia que tenemos de la vida es precisamente en tanto se lleva a cabo como mundo; el dasein mundea. El dasein es el único ente que es mundano, que tiene mundo. Empero, la nota distintiva es que él trasciende el mundo, no está en el mundo; él es quien abre el mundo.

En este sentido, cabe indagar por la relación que la vida fáctica establece con el mundo, es decir, la cuestión por la disposición afectiva (befindlichkeit) o la constitución ontológica del ahí. La disposición afectiva refiere primordialmente que existo y no solo existir sin más, sino el deber de existir. Ella es un modo de existencia fundamental en el cual se presenta el dasein como afectivamente determinado, dado que de alguna manera me antecede el hecho de tener que ser; la existencia me viene a mi ser en cuanto ser.

En el temple de ánimo (plano óntico de la disposición afectiva), el dasein ya está siempre afectivamente abierto como aquel ente al que la existencia le ha sido confiada en su ser, un ser que él tiene que ser existiendo (Heidegger, 1927, p. 139).

La disposición afectiva del dasein no procede de adentro ni de fuera, sino de manera más originaria, como modo de ser del dasein. Ella es el modo como el ser humano, en cuanto dasein, se instala afectivamente frente al mundo. Igualmente, se puede señalar que el estar afectivamente frente al mundo corresponde, en el orden ontológico, a lo designado con el concepto disposición afectiva; mientras que en el orden óntico es lo más conocido y cotidiano: el estado o temple de ánimo (stimmungen). Que anímicamente el dasein este templado implica el decir las maneras del cómo nos encontramos en la cotidianidad, por ende, este temple refiere que el dasein es apertura al mundo, empero, no implica apropiárselo reflexivamente ni un provocárselo deliberadamente. Los estados de ánimos nos dan la noticia de que hemos llegado tarde frente a aquello que ya está abierto. El dasein siempre va a estar afectivamente abierto al mundo o en su condición de arrojado ante su facticidad. Así, nos encontramos con el primer carácter ontológico: la disposición afectiva, a saber, que el dasein en su condición de arrojado (geworfenheit. estar arrojado/yecto), pero ella viene acompañada de una aversión esquivadora.

El estado de ánimo no abre mirando hacia la condición de arrojado, sino en la forma de una conversión o una aversión. De ordinario, el estado de ánimo no se vuelve hacia el carácter de carga que el dasein manifiesta en él, y menos aun cuando se encuentra liberado de esa carga en el estado de ánimo elevado. Esta aversión es siempre lo que es, en la forma de la disposición afectiva (Heidegger, 1927, pp. 139-140).

Esta forma de la disposición afectiva -la aversión- se podría explicar de la siguiente manera: la facticidad no es 
un hecho en neto (factum), sino el carácter de un ser del dasein asumido al mismo tiempo de la existencia; es el estado de yecto presenciado como el tener que ser siendo. El estar yecto es la imposibilidad de justificar mi existencia; es estar sin fundamento o indeterminado; es la imposibilidad de anticiparme a mi existencia; no la anticipo, no la escojo, simplemente existo y esto es lo que trato de esquivar. Por tanto, este carácter de sin fundamento (geworfenheit) me abre a la aversión esquivadora. Cabe señalar que todas las expresiones de ánimo tienen que ver con la verdad (stimmung: stimmen: calibrar/stimme: voz, relacionada con la idea de verdad) o lo que es lo mismo, la disposición afectiva es entendida como un modo verdadero de ser.

En lo anterior, se ha intentado señalar el cómo la aperturidad del ahi se expresa como poder-ser; empero, no se puede concluir que el poder ser se agota en befindlichkeit. Hay otras maneras como se puede tematizar el poder-ser; siendo así, el "comprender" (verstehen) también es un carácter de este poder-ser. "La aperturidad del ahí en el comprender es también una manera del poder-ser del dasein" (Heidegger, 1927, p. 150). Heidegger aleja el sentido de "comprender" del uso tradicional; ya no es entendido en su carácter de ejercicio epistemológico, es decir, es un "entender" que está lejos y antes de la proposición (Grondin, 1999). Comprender es un acto dado en el dasein mismo; es un existenciario en cuanto da un preentendimiento de las cosas del mundo vivencial; recordemos que el mundo se abre como horizonte de uso de las cosas en cuanto ellas están dadas en su para qué; las cosas del mundo son ejecutadas desde sus usos. Este comprender es un entender hermenéutico ya que ve las cosas (útiles) a partir de sus usos. No obstante, este comprender posee un carácter más originario: la preocupación por el sí mismo del ser-ahí. Esta preocupación es un experimentar el ser propio como única posibilidad, en donde el dasein abre frente a sí mismo su propio ser para saber lo que pasa consigo mismo. Este comprender, entonces, es el poder ser afectivamente dispuesto y enraizado a ciertas posibilidades; o mejor, su posibilidad original es su ser. Por tanto, "el comprender, escribe Heidegger, es el ser existencial del propio poder-ser del dasein mismo, de tal manera que este ser abre en sí mismo lo que pasa consigo mismo" (Heidegger, 1927, p. 148).

Pese a lo anterior, en la raíz del "comprender" aparece una problemática: el círculo vicioso, en cuanto "toda comprensión que haya de aportar comprensión deber haber comprendido ya lo que en ella se ha de interpretar" (Heidegger, 1927, p. 155). Si fuera cierto que la comprensión corresponde a un círculo vicioso que no es más que la correspondencia con el ideal científico del conocimiento, es porque la demostración científica no debe presuponer lo que ella tiene que demostrar. Este círculo vicioso -según el ideal científico- debe eliminarse si la historiografía quiere fundamentar su "significación espiritual". Para Heidegger esta interpretación es incorrecta; ella, mal comprende el comprender. "Sin embargo, ver en este círculo un circulus vitiosus y buscar cómo evitarlo, o por lo menos 'sentirlo' como imperfección inevitable, significa mal comprender radicalmente el comprender" (Heidegger, 1927, p. 156). Este no puede adecuarse al ideal de conocimiento como ejercicio de aprehender lo dado a la mano en su legítima incomprensibilidad. Lo importante, entonces, no es evitar el círculo hermenéutico, sino saber entrar en él, dado que este círculo no es un modo de conocimiento científico sino expresión de la estructura existencial del ser-ahí. Esta es la posibilidad originaria de conocimiento propio.

Hasta aquí se ha señalado que el dasein tiene ya siempre un cierto modo de relacionarse con el mundo y comprenderlo, modo que está atestiguado en la disposición afectiva y en la comprensión. Ahora, se puede comprender junto a ello la caída (verfallenheit) - determinación constitutiva del dasein-e introduzcamos la pregunta que hace Heidegger a la hora de tratar la totalidad ontológica del dasein, ya que hasta el momento todos los existenciarios se han presentado, en la analítica existenciaria, como separados: "Será posible captar en su totalidad este todo estructural de la cotidianidad del dasein?”). Cabe destacar que esta pregunta va acompañada de otras dos: "¿Será posible poner de tal manera unitariamente de relieve el ser del dasein que desde él se pueda comprender la esencial cooriginariedad de las estructuras mostradas y sus correspondientes posibilidades existenciales de modificación? ¿Hay alguna vía para llegar fenoménicamente a este ser, sobre la base del actual planteamiento de la analítica existenciaria?" (Heidegger, 1927, pp. 182-183)

La caída corresponde a la interpretación dada a la existencia inauténtica. "El dasein en cuanto cadente ha desertado ya de si mismo, entendido como fáctico estar-en-el-mundo, ha caído no en algo entitativo con lo que pudiera llegar o no llegar, quizás a tropezar en el transcurso de su ser, sino que ha caído en el mundo, en ese mismo mundo que forma parte de su ser" (Heidegger, 1927, p. 177).

El dasein puede ser auténtico o inauténtico, "puede, o bien 'elegirse', conquistarse, o bien perderse y no conquistarse en modo alguno" (Vattimo, 1987, p. 35). Igualmente, la inautenticidad parece caracterizarse por la incapacidad de alcanzar una verdadera comprensión, ya que "en lugar de encontrar la cosa misma se mantiene uno en las opiniones comunes" (Vattimo, 1987, p. 35). 
Entonces, ¿qué es la existencia autentica? Ella refiere al dasein como proyectarse "sobre la base de su posibilidad más suya" (Vattimo, 1987, p. 35); es decir, la existencia autentica seńala el dasein como capaz de apropiarse de sí y al mismo tiempo capaz de abrirse a las cosas.

Pero, ¿cómo introducir la pregunta por la totalidad estructural del dasein? La existencia, la facticidad y la caída, son los elementos estructurales del dasein conjugados en la comprensión del dasein como cura/cuidado (sorge). La sorge es una estructura existencial que corresponde al conjunto de disposiciones que constituyen el existir humano:

Un cierto mirar hacia delante, un atenerse a la situación en que ya se está, un habérselas con los entes en medio de los cuales uno se encuentra. En efecto, cuando se hace algo con "cuidado" se está vuelto hacia lo que viene en el futuro inmediato, hacia lo que hay que hacer. A la vez se está arraigado en la concretísima situación en la que ya nos movemos en cada caso. Además, en estas dos disposiciones se está en contacto con las cosas en medio de las cuales nos encontramos (Heidegger, 1927, p. 440).

La circularidad del trinomio estructural del dasein: la existencia, la facticidad y la caída, son posibles desde el fenómeno fundamental que es la sorge en sus tres momentos: pre-ser-se, ser-cabe, ser-ya-en-el-mundo. El pre-ser-se/ anticiparse-a-sí, recoge el rasgo de la anticipación que es el dasein como poder-ser en el proyectar. La anticipación recurre al significado ex-sistenz; la anticipación recurre al cómo a este ente le va su ser.

En el anticiparse-a-sí en cuanto estar vuelto hacia el más propio poder-ser, radica la condición ontológico-existencial de la posibilidad del ser libre para posibilidades existentivas propias. [...] En el anticiparse-a-sí, el "sí" mienta, pues, en este caso, el sí-mismo del uno-mismo (Heidegger, 1927, pp. 193-194).

El ser-cabe refiere al estar junto-a, ya que el ente se encuentra dentro del mundo. El dasein mundea, el dasein está junto-a...; empero, este estar "junto-a" refiere que ya se está "absorbido" por el mundo.

En este cadente estar en medio de... se acusa, explícita o implícitamente, comprendido o no comprendido, el huir ante la desazón que habitualmente queda oculta junto con la angustia latente, porque el carácter público del uno reprime todo lo que no es familiar. En el anticiparse-a-sí-estando-yaen-un-mundo se incluye de modo esencial el cadente estar en medio de lo intramundanamente a la mano de la ocupación (Heidegger, 1927, p. 193).

En definitiva, podemos concluir junto a Vattimo que:
Sorge es el ser del dasein, ya en su modalidad auténtica, ya en la modalidad inauténtica; pero esta última, como ha resultado claramente de los varios pasajes de la analítica existenciaría, no es más que el modo de ser parcial, derivado y deyecto de las estructuras auténticas; por eso el estudio del dasein en el modo en que se presenta en la cotidianidad media conduce empero al descubrimiento de las estructuras auténticas de la existencia (Vattimo, 1987, p. 38).

No obstante, cabe agregar que la unidad de estos tres momentos es posible por el tiempo; por tanto, "la proyección de un sentido del ser en general se puede llevar a cabo en el horizonte del tiempo" (Heidegger, 1927, p. 233). La unidad original de la estructura de la sorge reside en la temporalidad. Él posibilita la unidad de la existencia, la facticidad y la caída, constituyendo así la totalidad del dasein como estructura. La ex-sistencia y la temporalidad se co-pertenecen porque la esencia de la temporalidad es estar por fuera de sí, Auser-sich, quizá el más original; mientras que la ex-sistencia también era constituida por un "fuera de sî" que asumía sus más propias posibilidades. "Posibilidades no ajenas, sino propias: en y para la existencia misma" (Navarro-Cordón, 1966, p. 34).

Si la temporeidad constituye el sentido originario del ser del dasein y si a este ente en su ser le va este mismo ser, entonces el cuidado tendrá que hacer uso del "tiempo" y por consiguiente, contar con "el tiempo". La temporeidad del dasein desarrolla el "cómputo del tiempo". El "tiempo" -experimentado en este- es el aspecto fenoménico inmediato de la temporeidad. De él brota la comprensión cotidiana y vulgar del tiempo. Y esta se despliega en el concepto tradicional del tiempo. El esclarecimiento del origen del "tiempo" "en el que" comparece el ente intramundano, del tiempo como intratemporeidad, pone de manifiesto una esencial posibilidad de temporización de la temporeidad. Con esto se prepara la comprensión para una temporización incluso más originaria de la temporeidad. En ella se funda la comprensión del ser que es constitutiva del ser del dasein (Heidegger, 1927, pp. 232-233).

\section{Conclusión}

Indagar por los rasgos de la ontología fundamental es una tarea titánica, por lo que sería fácil afirmar que no existen tales rasgos. Empero, ello se debe al modo de ingreso a la temática; o, quizá, la metodología empleada por el mismo Heidegger dificulte tal labor. La metodología de Heidegger es radical. Él acusa a la tradición filosófica de olvidar la pregunta por el ser y por ende, la rescata; crea nuevos conceptos ya que los existentes no serán eficaces o estarán sesgados para el análisis; y cada vez que parece 
llegar a una conclusión, la deconstruye hasta el punto de presentarla casi nueva.

Se puede rastrear la ontología fundamental en la radicalidad del pensamiento heideggeriano; luego, en la analítica existenciaría del dasein, que no es otra cosa que la descripción fenomenológica del dasein. Sin embargo, hay que indicar que esta no agota aquella; es decir, aunque la analítica existenciaría explicite la ontología fundamental, no la agota. Y finalmente, se puede seguir en la superación de la metafísica, pero tal tarea no se abordó aquí; quizá se introdujo en el sentido de clarificar -aunque no directamente- que la metafísica no desaparece.

En suma, si bien rastrear los rasgos de la ontología fundamental es una labor ardua, no se puede concluir necesariamente que no existan dichos rasgos. Por el contario, en ello radica lo fundamental de esta ontología: es una nueva filosofía, desde sus conceptos hasta su metodología. Si la ontología fundamental tiene como primer rasgo la diferencia ontológica y ya que ella es un logro de la analítica existenciaría del dasein, es necesaria, por tanto, su explicitación.

Estas son las premisas que hemos logrado: a) la pregunta por el ser tematiza la diferencia ontológica, es decir, la relación entre el ser y el ente dada en el dasein; b) el dasein posee primacía óntico-ontológica en cuanto es existente y está determinado por ella; $\mathrm{y}$ ante el problema de acceso a dicho ente se presenta la cotidianidad como solución; c) la ex-sistencia corresponde a la apertura del dasein hacia el horizonte de la posibilidad; es decir, el dasein en cuanto existente, es poder-ser. Además, dado que el dasein se mueve en una precomprensión de la existencia es imposible pensarlo sin mundo, ya que esa precomprensión es un reflejo de la constitución ontológica del mundo. El dasein en cuanto existe, mundea. El dasein es ser-en-el-mundo; d) al ganar la comprensión de dasein como ser-en-el-mundo, es necesario explicitar sus existenciarios (sus modos de ser). El primero es la disposición afectiva, que refiere la relación que la vida fáctica establece con el mundo; el segundo, es la comprensión; aunque se puede señalar por razones de orden metodológico, que él está antes de la disposición afectiva, ya que "el dasein está en el mundo ante todo y fundamentalmente como comprensión antes que como afectividad" (Vattimo, 1987, p. 25); e) la caída juega el papel de existenciario del dasein y con ella, la autenticidad o inautenticidad del dasein; autentico, en cuanto toma su propio ser como posibilidad más propia; inauténtico, si olvida su ser como su posibilidad más propia; f) la unidad estructural, la totalidad del dasein, exige ser tomada como tal. El dasein no solo es existencia, facticidad o caída; es todas al mismo tiempo. La sorge (cura/cuidado) cumple está función. En ella se conjugan las divergencias y convergencias de las tres. En definitiva, la sorge es el dasein que asume la existencia auténticamente. Empero, como ella viene determinada por el tiempo, el dasein es tal en cuanto temporalidad. Tan solo en la temporalidad del dasein se puede comprender el sentido general del ser. No obstante, ¿cómo sostener que la ontología fundamental es la superación de la metafísica; o lo que es lo mismo, que ella busca el fundamento de la metafísica?

\section{Referencias}

García-Ruiz, P. E. (2002).¿Ontología fundamental o teoría del conocimiento? Heidegger crítico del neokantismo. Signos filosóficos, 7, pp. 125-150.

Grondin, J. (1999). Introducción a la hermenéutica filosófica. Barcelona: Herder.

Heidegger, M. (1927). Sery tiempo. Santiago de Chile: Editorial Universitaria.

Heidegger, M. (1989). Contribuciones a la filosofia. (Del acontecimiento). Valparaíso: Universidad Católica de Chile.

Heidegger, M. (2008). jAlma mía! Cartas a su mujer Elfride 1915-1970. Buenos Aires: Manantial.

Held, K. (1997) El camino de Heidegger a las cosas mismas. Franciscanum: revista de las ciencias del espíritu, Vol. 34, 115. pp. 7-24.

Lambert-Ortiz, C. (2008) "Heidegger y el proyecto de una ciencia originaria”. Veritas: revista de filosofia y teología, 18 (3), pp. 87-97.

Magallon-Anaya, M. (1991). Dialéctica de la filosofía latinoamericana. Una filosofía en la historia. México: Universidad Nacional Autónoma de México.

Navarro-Cordón, J. M. (1966) Sentido de la ontología fundamental en Heidegger. Logos. Anales del seminario de metafisica, 1, 29-52.

Rivera, J. E., \& Stuven, M. T. (2008). Comentario a ser y tiempo de Martin Heidegger. Santiago de Chile: Ediciones Universidad Católica de Chile.

Strauss L. (1989). The Rebirth of Classical Political Rationalism: an introduction to the Thoug of Leo Strauss. Chicago: Chicago Press.

Vattimo, G. (1987, Traducción de A. Báez) Introducción a Heidegger. México, D.F.: Gedisa.

Xolocotzi-Yáñez, Á. (2011) Una crónica de Ser y tiempo de Martin Heidegger. Puebla: Ítaca, Benemérita Universidad Autónoma de Puebla.

$102<$ Universidad de San Buenaventura, Cali - Colombia 